\title{
The expression of circRNAs as a promising biomarker in the diagnosis and prognosis of human cancers: a systematic review and meta-analysis
}

\author{
Han-Xi Ding ${ }^{1}$, Zhi Lv ${ }^{1}$, Yuan Yuan ${ }^{1,2}$ and Qian Xut ${ }^{1,2}$ \\ ${ }^{1}$ Tumor Etiology and Screening Department of Cancer Institute and General Surgery, The First Affiliated Hospital of China \\ Medical University, Key Laboratory of Cancer Etiology and Prevention, China Medical University, Liaoning Provincial Education \\ Department, Shenyang 110001, China \\ ${ }^{2}$ National Clinical Research Center for Digestive Diseases, Xi'an 110001, China \\ Correspondence to: Qian Xu, email: qxu@cmu.edu.cn \\ Yuan Yuan, email: yuanyuan@cmu.edu.cn \\ Keywords: diagnosis, prognosis, cancers, circRNAs, biomarker
}

Received: August 02, $2017 \quad$ Accepted: October 05, $2017 \quad$ Published: December 15, 2017

Copyright: Ding et al. This is an open-access article distributed under the terms of the Creative Commons Attribution License 3.0 (CC BY 3.0), which permits unrestricted use, distribution, and reproduction in any medium, provided the original author and source are credited.

\section{ABSTRACT}

Background: CircRNAs, a type of non-coding RNAs with special loop structure, of which the aberrant expression is closely related to tumor growth, proliferation, metastasis and recurrence. It remains unclear whether they have the potential to be biomarkers for diagnosis and prognosis of cancers. The study aims to clarify the relationship of circRNAs expression with cancers diagnosis and prognosis.

Materials and Methods: Sensitivity, specificity, area under curve (AUC) and receiver operating characteristic curve (ROC) were calculated to evaluate the diagnostic efficacy; Hazard ratio (HR) of overall survival (OS), disease free survival (DFS) and recurrence free survival (RFS) were calculated to evaluate the association between circRNAs expression and survival of cancer patients.

Results: A total of $\mathbf{2 7}$ studies were involved in the meta-analysis, including 16 diagnostic and 11 prognostic articles. Among the diagnostic studies, 18 kinds of circRNAs had been investigated, in which 3 were up regulated and 15 were down regulated. Their pooled sensitivity, specificity and AUC were $0.71(0.65-0.77)$, $0.77(0.72-0.81)$ and $0.81(0.77-0.84)$, respectively. In stratified analysis, a higher specificity was shown in circRNAs for diagnosing gastric cancer and hepatocellular cancer. 12 circRNAs were involved in the prognostic studies, including 6 up-regulated and 6 down-regulated circRNAs. Their overall HR of OS and DFS/RFS were 1.37(0.98$1.75)$ and $2.28(0.77-3.79)$, respectively.

Conclusions: CircRNAs have the potential to be biomarkers for diagnosis and prognosis of cancers. Further investigations are still needed to explore the clinical value of circRNAs as tumor markers.

\section{INTRODUCTION}

Circular RNAs (circRNAs) are formed by the covalent binding between phosphodiester bonds on their 3 ' and 5' ends, which are distinct from linear RNAs [1-3]. Due to the lacking of free ends, circRNAs could escape the effects from exonuclease and ribonuclease, thus they are more stable than linear RNAs in cells [4]. So far, about one hundred thousand circRNAs have been identified which exert extensive functions in human body such as miRNA sponges and gene regulator [5-8]. There has been mounting evidence that circRNAs play significant roles in tumor genesis, malignant transformation, signal transduction, invasion, metastasis and angiogenesis. For example, circ 100284 could up-regulate the expression of target gene EZH2 by inhibiting miR-217, elevate the concentration of cyclin D1, promote the cell cycle and induce vicious transformation of cells [9]; circ-ITCH may lead to cell cycle arrest and malignant cells suppression by 
affecting the Wnt signal pathway [10]; circ-Foxo3 could inhibit tumor angiogenesis [11]; ciRS-7 is closely related to hepatic microvascular invasion (MVI) by modulating the expression of miR-7 as well as its target genes, PIK3CD and p70S6K [12]. It has been found that circRNAs expression is highly stable in saliva, blood and exosomes, which could be attributed to the effective mechanisms of their synthesis and elimination in cells [13-15]. Moreover, circRNAs are relatively abundant both in cells and extracellular fluids with a long half-time period $[13,16]$. As a result, they are very likely to be biomarkers for cancer diagnosis and prognosis which could provide a promising method for clinical practice $[1,3]$.

Although, in recent years, some certain circRNAs have been reported to act as stable markers for diagnosis and prognosis of cancer, there still are some questions affecting the evaluation of circRNAs in cancer diagnosis and prognosis, including limited number of research cases, skimble-scamble sample source and disease status, various experiment methods and other uncontrolled factors. Therefore, the current research data about the clinic role of circRNAs remains unconvincing. Accordingly, we conducted a systematic review and meta-analysis on the association of circRNAs expression with cancer diagnosis and prognosis for the first time. The study aims to clarify their relationship and the possibility of circRNAs as tumor markers, which could be helpful for clinical decision-making and the development of circRNAs-based targeted therapy.

\section{RESULTS}

\section{Selection of studies}

A total of 1905 records were retrieved initially from databases, and 27 articles were involved in our final meta-analysis after multiple steps of selection (Figure 1) [12, 17-42]. Among the enrolled studies, 16 were related to diagnosis [17, 19-24, 26, 28, 29, 34-39], and the others were about prognosis [12, 18, 25, 27, 30-33, 40-42]. These studies referred to 30 kinds of circRNAs in all, 3 of which were focused on the combined effects (four circRNAs: hsa_circRNA_101308, hsa_circRNA_104423, hsa_circRNA_104916, hsa_circRNA_100269; three circRNAs: hsa_circRNA_10219, hsa_circRNA_006054, hsa_circRNA_406697; and two circRNAs: hsa circRNA_0007874, hsa_circRNA_104135).

\section{Diagnostic meta-analysis of circRNAs in cancers}

\section{Study characteristics and quality assessment}

The main characteristics of diagnostic studies were shown in Table 1. Sixteen studies including 1735 cases and 1707 controls were enrolled in the diagnostic metaanalysis. They were all published between February 2015 and September 2017. The main detection method for circRNAs expression was quantitative real-time reverse transcription PCR (qRT-PCR), while only one study applied fluorescence in situ hybridization (FISH). Samples in most researches were selected from cancerous and paracancerous tissues taken from surgery, while circRNAs expression in plasma was only detected by a single study. Quality assessment of diagnostic accuracy studies-2 (QUADAS-2) was employed to evaluate the quality of enrolled diagnostic studies. All of them were suggested to have moderate to high quality and thus appropriate for meta-analysis (Supplementary Table 1).

\section{Meta-analysis findings}

Among the 18 diagnosis-related circRNAs, 3 was up-regulated (hsa_circ_0005075, hsa_circ_0013958, circCCDC66) and 15 were down-regulated (hsa circ_002059, hsa_circ_001988, hsa_circ_0001649, hsa circ_0000190, hsa_circ_0004018, hsa_circ_0001895, circZKSCAN1, hsa_circ_103809, hsa_circ_104700, hsa_circ_003570, hsa_circ_0014717 hsa_circ_100219, hsa_circ_006054, hsa_circ_406697, hsa_circ_0006633, Table 2, Table 3). To explore whether circRNAs could serve as effective markers for cancer diagnosis, we calculated the overall sensitivity, specificity and diagnostic odds ratio (DOR), which were $0.71(0.65$ $0.77), 0.77(0.72-0.81)$ and 8.37(6.14-11.39), respectively (Figure 2). The summary receiver operator characteristic curve (SROC) was shown in Supplementary Figure 1 and the corresponding AUC was 0.81(0.77-0.84), suggesting a relatively high accuracy of circRNAs for cancer diagnosis.

\section{Subgroup and meta-regression analysis}

Stratified analysis was performed based on sample size (>100 vs. $<100)$ and cancer type (Gastric cancer vs. Colorectal cancer vs. Hepatocellular cancer). In the subgroup with large sample size $(>100)$, the pooled sensitivity, specificity and AUC were 0.71(0.63-0.78), $0.76(0.52-0.72)$ and $0.77(0.73-0.80)$; while $0.74(0.66-$ $0.80), 0.84(0.75-0.90)$ and $0.78(0.74-0.82)$ for small sample size $(<100)$. The pooled sensitivity, specificity and AUC in the subgroup of gastric cancer were $0.66(0.57-$ $0.74), \quad 0.80(0.72-0.85)$ and $0.80(0.78-0.83)$; while $0.72(0.60-0.82), 0.67(0.58-0.76)$ and $0.76(0.72-0.79)$ for colorectal cancer and $0.73(0.59-0.83), 0.79(0.72-0.85)$, $0.86(0.83-0.89)$ for hepatocellular cancer, respectively (Figure 3, Figure 4, Table 4).

Meta-regression analysis for the subgroups was next conducted. Both the $P$ values for sample size and cancer type were $>0.10$, suggesting no significant impact of subgroups on the pooled results.

\section{Sensitivity analysis and publication bias}

Sensitivity analysis was performed to explore the influence of an individual study on the pooled results. No significant change was observed when compared with previous results after removal of each study (Supplementary Figure 2). The threshold effect was also evaluated, which was derived from the differences 
between sensitivity and specificity. Their Spearman correlation coefficient was -0.52 and $P=0.270$, indicating no heterogeneity from threshold effect and thus reliability of our results.

Deek's plot was employed to assess the publication bias. Significant publication bias was shown in the study ( $\mathrm{t}=3.06$ and $P=0.007$, Supplementary Figure 2), suggesting that only researches with positive findings were published or accepted.

\section{Prognostic meta-analysis of circRNAs in cancers}

\section{Study characteristics and quality assessment}

Fifteen records were enrolled in the prognostic meta-analysis, including 11 studies with 1891 samples in all ( 7 for gastric cancer, 2 for colorectal cancer, 3 for hepatocellular cancer, 1 for non-small cell lung cancer and 1 for breast cancer). Among them, two articles were focused on disease free survival (DFS) and recurrence free survival (RFS); eight were focused on overall survival (OS); the other one was related to both DFS and OS. The main characteristics of prognostic studies were shown in Table 2. All the samples were selected from Asian tissue. The major detection method for circRNAs expression was quantitative real-time reverse transcription PCR (qRTPCR), while only one study applied fluorescence in situ hybridization (FISH). Newcastle-Ottawa Scale (NOS) was employed to evaluate the quality of enrolled studies, and they were all suggested to be appropriate for meta-analysis (Supplementary Table 2).

\section{Meta-analysis findings}

Among the 12 prognosis-related circRNAs, 6 were up-regulated (circPVT1, ciRS-7, hsa_circ_101308, hsa circ_100876, circRNA-MYLK, circRNA_104135) and $\overline{6}$ were down-regulated (hsa_circ_104423, hsa_circ_104916, hsa_circ_100269, hsa_circ_0007874, hsa_circ_104135, circ ITCH Table 2, Table 3 ). It was shown that the overall HR with $95 \%$ CI for circRNAs expression in caner prognosis was 1.37(0.98-1.75) (Table 5, Figure 5), suggesting poor potentials of circRNAs expression to become biomarkers in OS prediction for cancer patients. Furthermore, the association between circRNAs expression and DFS/RFS was analyzed, and its HR with 95\% CI was 2.28(0.77-3.79) (Table 5, Figure 5), also suggesting negative prospects for circRNAs expression to be applied to prediction in DFS/RFS of cancer patients.

\section{Subgroup and meta-regression analysis}

Stratified analysis for OS was performed next. With respect to OS, the HRs with 95\% CIs for up-regulated circRNAs and down-regulated circRNAs were 1.85(1.262.44 ) and 0.46(0.32-0.59), respectively (Table 6, Figure 6).

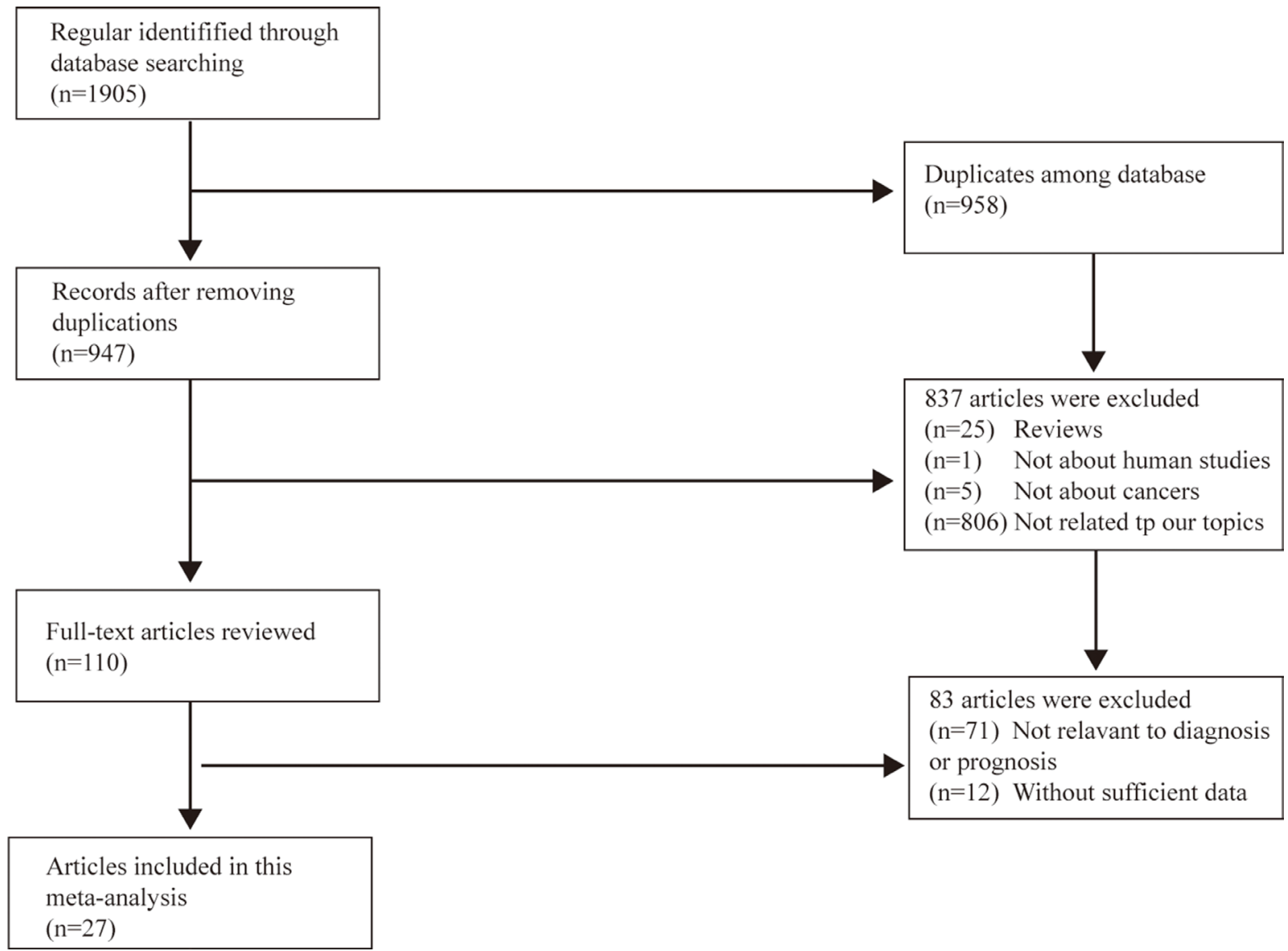

Figure 1: Flow diagram of the study selection process. 
Table 1: The main featurs of the included studies for diagnostic meta-analysis

\begin{tabular}{|c|c|c|c|c|c|c|c|c|c|c|c|c|c|}
\hline $\begin{array}{l}\text { Reference } \\
\text { number }\end{array}$ & Auhor & Year & cirRNAs & Country & Ethnicity & $\begin{array}{c}\text { Cancer } \\
\text { type }\end{array}$ & $\begin{array}{c}\text { Case/ } \\
\text { Control }\end{array}$ & Sample & AUC & $\mathrm{Se}$ & Sp & $\begin{array}{c}\text { Detection } \\
\text { methods }\end{array}$ & Citation \\
\hline 1 & Peifei Li et al & 2015 & hsa_circ_002059 & China & Asian & GC & $101 / 101$ & tissue & 0.730 & 0.810 & 0.620 & qRT-PCR & 24 \\
\hline 2 & $\begin{array}{c}\text { Xuning Wang } \\
\text { et al }\end{array}$ & 2015 & hsa_circ_001988 & China & Asian & $\mathrm{CRC}$ & $31 / 31$ & tissue & 0.788 & 0.680 & 0.730 & qRT-PCR & 20 \\
\hline 3 & Meilin Qin et al & 2016 & hsa_circ_0001649 & China & Asian & $\mathrm{HCC}$ & $89 / 89$ & tissue & 0.630 & 0.810 & 0.690 & qRT-PCR & 26 \\
\hline 4 & $\begin{array}{c}\text { Xingchen Shang } \\
\text { et al }\end{array}$ & 2016 & hsa_circ_0005075 & China & Asian & $\mathrm{HCC}$ & $30 / 30$ & tissue & 0.940 & 0.833 & 0.900 & qRT-PCR & 21 \\
\hline 5 & Shijun Chen et al & 2017 & hsa_circ_0000190 & China & Asian & GC & $104 / 104$ & tissue & 0.750 & 0.721 & 0.683 & qRT-PCR & 23 \\
\hline 6 & Shijun Chen et al & 2017 & hsa_circ_0000190 & China & Asian & GC & $104 / 104$ & plasma & 0.600 & 0.414 & 0.875 & qRT-PCR & 23 \\
\hline 7 & Liyun Fu et al & 2017 & hsa_circ_0004018 & China & Asian & $\mathrm{HCC}$ & $102 / 129$ & tissue & 0.848 & 0.716 & 0.815 & qRT-PCR & 17 \\
\hline 8 & Wen-han Li et al & 2017 & hsa circ 0001649 & China & Asian & GC & $76 / 76$ & tissue & 0.834 & 0.711 & 0.816 & qRT-PCR & 22 \\
\hline 9 & Yongfu Shao et al & 2017 & hsa_circ_0001895 & China & Asian & GC & $96 / 96$ & tissue & 0.792 & 0.678 & 0.857 & qRT-PCR & 29 \\
\hline 10 & $\begin{array}{c}\text { Zhicheng Yao } \\
\text { et al }\end{array}$ & 2017 & circZKSCAN1 & China & Asian & $\mathrm{HCC}$ & $102 / 102$ & tissue & 0.834 & 0.822 & 0.724 & FISH & 19 \\
\hline 11 & Peili Zhang et al & 2017 & hsa_circRNA_103809 & China & Asian & $\mathrm{CRC}$ & $170 / 170$ & tissue & 0.669 & 0.662 & 0.690 & qRT-PCR & 28 \\
\hline 12 & Peili Zhang et al & 2017 & hsa_circRNA_104700 & China & Asian & $\mathrm{CRC}$ & $170 / 170$ & tissue & 0.616 & 0.682 & 0.532 & qRT-PCR & 28 \\
\hline 13 & Liyun Fu et al & 2017 & hsa_circ_0003570 & China & Asian & $\mathrm{HCC}$ & $107 / 107$ & tissue & 0.700 & 0.449 & 0.868 & qRT-PCR & 39 \\
\hline 14 & Yongfu Shao et al & 2017 & hsa_circ_0014717 & China & Asian & $\mathrm{GC}$ & $96 / 96$ & tissue & 0.696 & 0.594 & 0.813 & qRT-PCR & 37 \\
\hline 15 & Xiaoli Zhu et al & 2017 & hsa_circ_0013958 & China & Asian & LAC & $49 / 49$ & tissue & 0.815 & 0.755 & 0.796 & qRT-PCR & 34 \\
\hline 16 & Xiaoli Zhu et al & 2017 & hsa_circ_0013958 & China & Asian & LAC & $30 / 30$ & plasma & 0.794 & 0.667 & 0.933 & qRT-PCR & 34 \\
\hline 17 & $\begin{array}{l}\text { Lingshuang Lü } \\
\text { et al }\end{array}$ & 2017 & $\begin{array}{c}\text { hsa_circ_100219,hsa_ } \\
\text { circ_006054,hsa_ } \\
\text { circ_406697 }\end{array}$ & China & Asian & $\mathrm{BrC}$ & $51 / 51$ & tissue & 0.820 & 0.825 & 0.732 & qRT-PCR & 36 \\
\hline 18 & Rongdan Lu et al & 2017 & hsa_circ_0006633 & China & Asian & GC & $96 / 96$ & tissue & 0.741 & 0.600 & 0.810 & qRT-PCR & 35 \\
\hline 19 & $\begin{array}{c}\text { Kuei-Yang Hsiao } \\
\text { et al }\end{array}$ & 2017 & circCCDC66 & China & Asian & CRC & $131 / 76$ & tissue & 0.884 & 0.927 & 0.740 & qRT-PCR & 38 \\
\hline
\end{tabular}

$\mathrm{GC}=$ Gastric Cancer; HCC=Hepatocellular Carcinoma; $\mathrm{CRC}=$ Colorectal Cancer; NSCLC=Non Small Cell Lung Cancer; LAC: Lung Adenocarcinoma; BC: Breast cancer; $\mathrm{AUC}=$ Area Under Curve; Se=Sensitivity; $\mathrm{Sp}=$ Specificity; qRT-PCR=Quantitative real time reverse transcription PCR; FISH=fluorescence in situ hybridization.
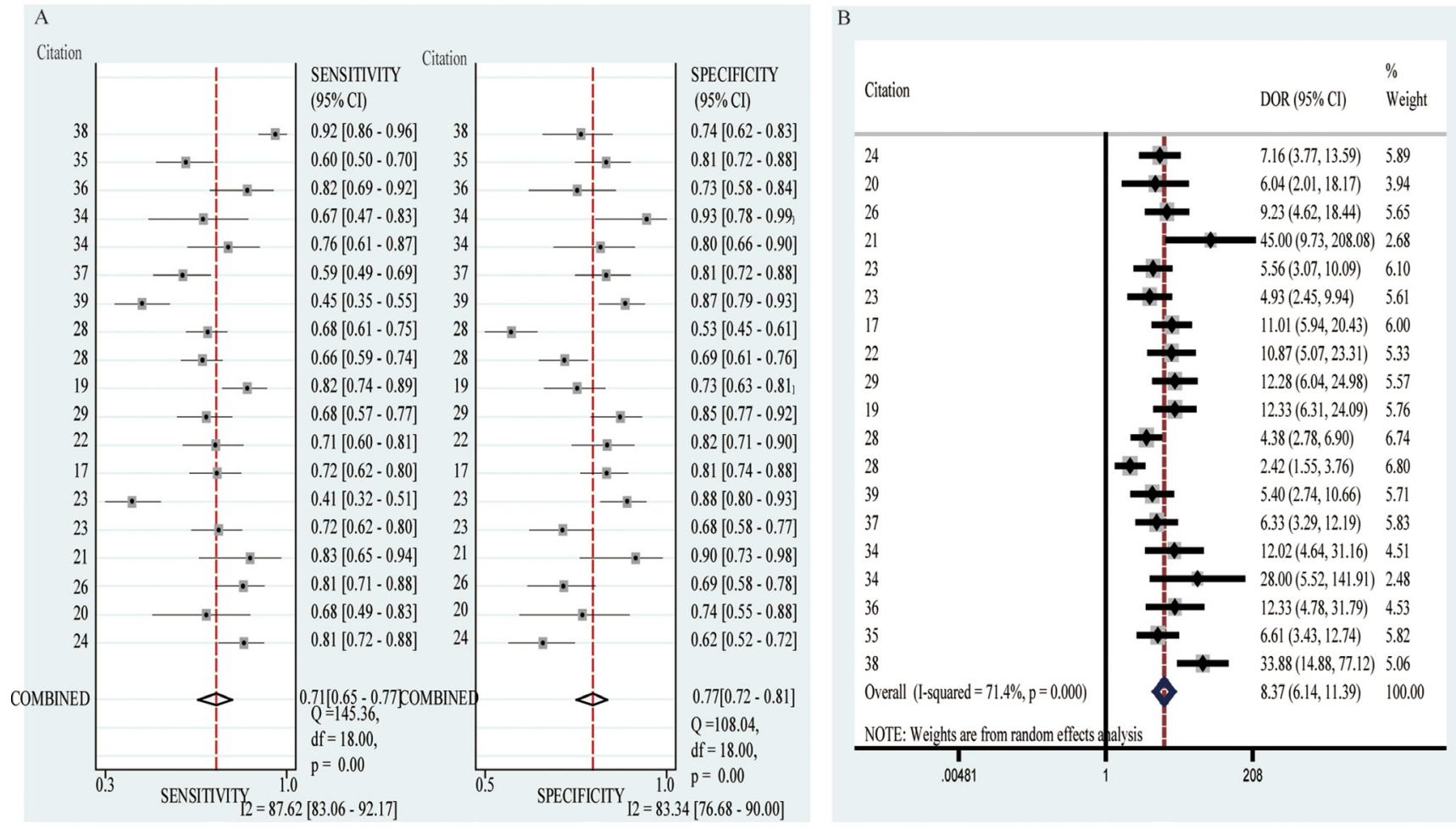

Figure 2: Forest plots of sensitivity and specificity and DOR value of diagnostic articles. (A) Forest plots of sensitivity and specificity of diagnostic articles. (B) The DOR value of diagnostic articles. 
Table 2: The main features of the included studies for prognostic meta-analysis

\begin{tabular}{|c|c|c|c|c|c|c|c|c|c|c|c|c|c|c|}
\hline $\begin{array}{c}\text { Referrence } \\
\text { number }\end{array}$ & Author & Year & circRNAs & Country & Ethnicity & Cancer & Sample & $\mathbf{N}$ & Stage & Survival & $\begin{array}{c}\text { Follow-up } \\
\text { (months) }\end{array}$ & HR( $95 \% C I)$ & $\begin{array}{c}\text { Detection } \\
\text { methods }\end{array}$ & Citation \\
\hline 1 & $\begin{array}{l}\text { Jie Chen } \\
\text { et al }\end{array}$ & 2017 & circPVT1 & China & Asian & GC & Tissue & 187 & I-IV & DFS & 85 & $0.490(0.330-0.720)$ & qRT-PCR & 30 \\
\hline 2 & $\begin{array}{c}\text { Liangliang } \\
\text { Xu et al }\end{array}$ & 2017 & ciRS7 (Cdr1as) & China & Asian & $\mathrm{HCC}$ & Tissue & 95 & I-IV & DFS & 63 & $1.450(0.870-2.410)$ & qRTPCR & 12 \\
\hline 3 & $\begin{array}{c}\text { Yan Zhang } \\
\text { et al }\end{array}$ & 2017 & $\begin{array}{l}\text { hsa_circRNA_101308, } \\
\text { hsa_circRNA_104423, } \\
\text { hsa_circRNA_104916, } \\
\text { hsa_circRNA_100269 }\end{array}$ & China & Asian & GC & Tissue & 67 & III & RFS & 12 & $6.248(2.534-15.403)$ & qRT-PCR & 25 \\
\hline 4 & $\begin{array}{c}\text { Yan Zhang } \\
\text { et al }\end{array}$ & 2017 & $\begin{array}{l}\text { hsa_circRNA_101308, } \\
\text { hsa_circRNA_104423, } \\
\text { hsa_circRNA_104916, } \\
\text { hsa_circRNA_100269 }\end{array}$ & China & Asian & GC & Tissue & 52 & III & RFS & 12 & $4.886(1.375-17.359)$ & qRT-PCR & 25 \\
\hline 5 & $\begin{array}{l}\text { Jie Chen } \\
\text { et al }\end{array}$ & 2017 & circPVT1 & China & Asian & $\mathrm{GC}$ & Tissue & 187 & I-IV & os & 83 & $0.600(0.400-0.880)$ & qRT-PCR & 30 \\
\hline 6 & $\begin{array}{c}\text { Wenhao } \\
\text { Weng et al }\end{array}$ & 2017 & ciRS-7 - A & China & Asian & $\mathrm{CRC}$ & Tissue & 153 & I-IV & OS & 100 & $2.070(1.098-3.902)$ & qRT-PCR & 18 \\
\hline 7 & $\begin{array}{l}\text { Wenhao } \\
\text { Weng et al }\end{array}$ & 2017 & ciRS-7 - A & Japan & Asian & $\mathrm{CRC}$ & Tissue & 165 & I-IV & OS & 133 & $2.690(1.257-5.741)$ & qRT-PCR & 18 \\
\hline 8 & $\begin{array}{l}\text { Jun-Tao } \\
\text { Yao et al }\end{array}$ & 2017 & hsa_circRNA_100876 & China & Asian & NSCLC & Tissue & 101 & I-IV & OS & 41 & $1.000(0.960-1.040)$ & qRT-PCR & 27 \\
\hline 9 & $\begin{array}{c}\text { Yan Zhang } \\
\text { et al }\end{array}$ & 2017 & hsa_circRNA_100269 & China & Asian & $\mathrm{GC}$ & Tissue & 112 & III & OS & 50 & $0.600(0.350-1.020)$ & qRT-PCR & 33 \\
\hline 10 & $\begin{array}{c}\text { Dan Han } \\
\text { et al }\end{array}$ & 2017 & $\begin{array}{c}\text { circMTO1 (hsa } \\
\text { circRNA_0007874/ } \\
\text { hsa_circRNA_104135) }\end{array}$ & China & Asian & $\mathrm{HCC}$ & Tissue & 116 & I-IV & OS & 80 & $0.340(0.220-0.510)$ & FISH & 42 \\
\hline 11 & $\begin{array}{l}\text { Zhenyu } \\
\text { Zhong } \\
\text { et al }\end{array}$ & 2017 & circRNA-MYLK & China & Asian & $\mathrm{BC}$ & Tissue & 32 & I-IV & OS & 43 & $3.920(1.900-8.100)$ & qRT-PCR & 31 \\
\hline 12 & $\begin{array}{c}\text { Xiu-Yan } \\
\text { Huang } \\
\text { et al }\end{array}$ & 2017 & hsa_circRNA_100338 & China & Asian & $\mathrm{HCC}$ & Tissue & 80 & I-IV & os & 126 & $1.000(0.970-1.03)$ & qRT-PCR & 40 \\
\hline 13 & $\begin{array}{l}\text { Haiyan Pan } \\
\quad \text { et al }\end{array}$ & 2017 & ciRS-7 & China & Asian & $\mathrm{GC}$ & Tissue & 102 & I-IV & OS & 60 & $2.110(0.940-3.890)$ & qRT-PCR & 32 \\
\hline 14 & $\begin{array}{l}\text { Haiyan Pan } \\
\text { et al }\end{array}$ & 2017 & ciRS-7 & China & Asian & GC & Tissue & 154 & I-IV & OS & 60 & $2.630(1.230-5.550)$ & qRT-PCR & 32 \\
\hline 15 & $\begin{array}{l}\text { Wenzhi } \\
\text { Guo et al }\end{array}$ & 2017 & circ-ITCH & China & Asian & $\mathrm{HCC}$ & Tissue & 288 & I-IV & OS & 90 & $0.450(0.290-0.680)$ & qRT-PCR & 41 \\
\hline
\end{tabular}

$\mathrm{GC}=$ Gastric Cancer; $\mathrm{HCC}=$ Hepatocellular Carcinoma; $\mathrm{CRC}=$ Colorectal Cancer; NSCLC $=$ Non Small Cell Lung Cancer; $\mathrm{BC}=\mathrm{Bladder}$ Cancer; $\mathrm{N}=$ number of cases; DFS=Disease

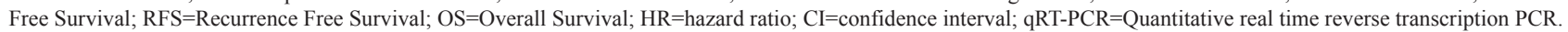
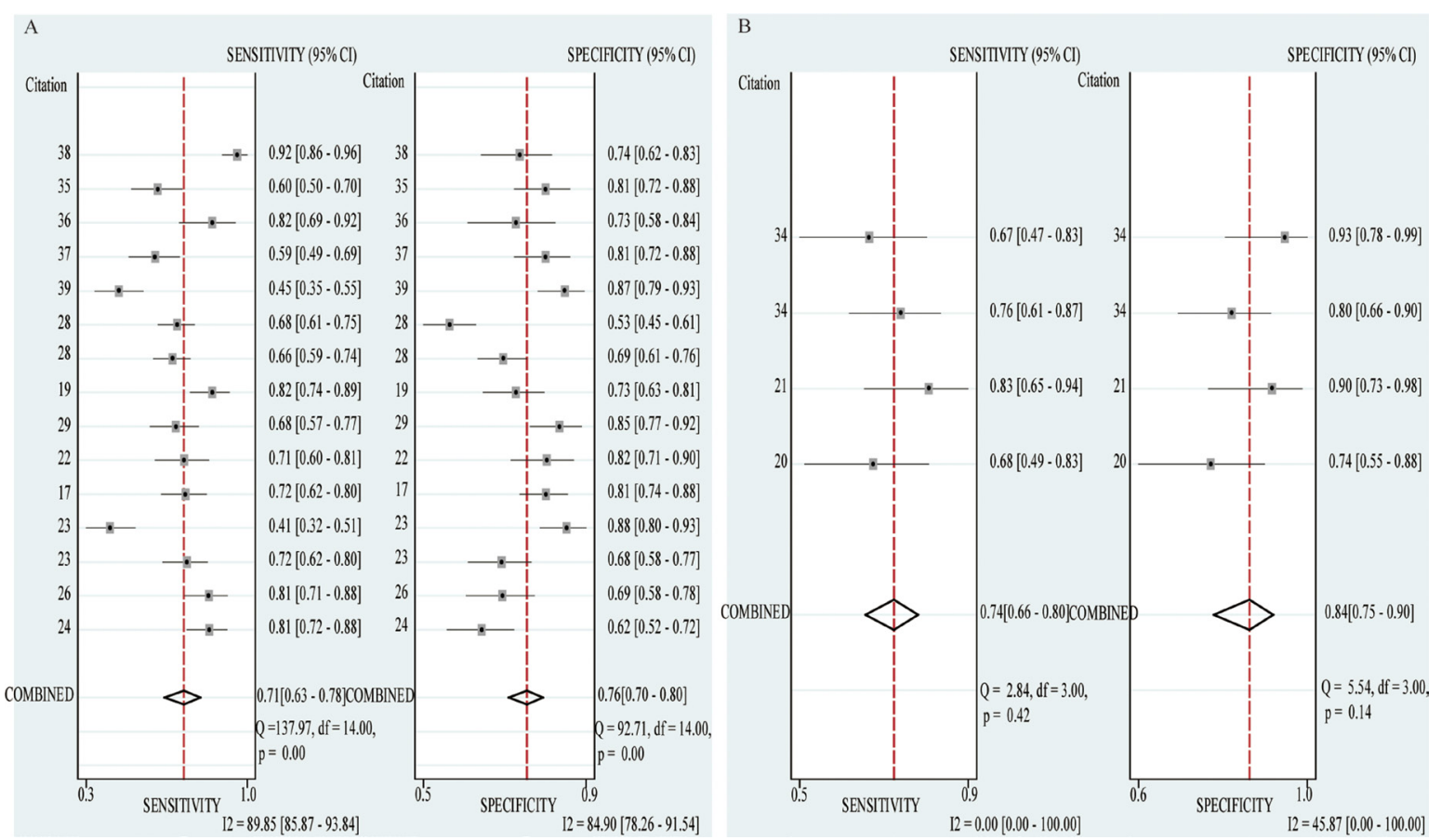

Figure 3: Forest plots of sensitivity and specificity of diagnostic articles in subgroup analysis. (A) Forest plots of sample size $>100$ subgroup. (B) Forest plots of sample size $<100$ subgroup. 
Table 3: CircRNAs and roles in cancers

\begin{tabular}{|c|c|c|c|c|c|c|}
\hline $\begin{array}{l}\text { Reference } \\
\text { number }\end{array}$ & CircRNAs & Prognosis & Role & $\begin{array}{c}\text { Cancer } \\
\text { Type }\end{array}$ & Function & Citation \\
\hline 1 & hsa_circ_002059 & Down-regulation & Suppressor & $\mathrm{GC}$ & Metastasis & 24 \\
\hline 2 & hsa_circ_001988 & Down-regulation & Suppressor & $\mathrm{CRC}$ & Invasion/Differentiation & 20 \\
\hline 3 & hsa_circ_0001649 & Down-regulation & Suppressor & $\mathrm{HCC}$ & $\begin{array}{l}\text { Development/ } \\
\text { Progression }\end{array}$ & 26 \\
\hline 4 & hsa_circ_0000190 & Down-regulation & Suppressor & $\mathrm{GC}$ & $\begin{array}{l}\text { Occurrence/ } \\
\text { Progression }\end{array}$ & 23 \\
\hline 5 & hsa_circ_0004018 & Down-regulation & Suppressor & $\mathrm{HCC}$ & Occurrence/Metastasis & 17 \\
\hline 6 & hsa circ 0001649 & Down-regulation & Suppressor & $\mathrm{GC}$ & Differentiation & 22 \\
\hline 7 & hsa_circ_0001895 & Down-regulation & Suppressor & $\mathrm{GC}$ & Occurrence & 29 \\
\hline 8 & circZKSCAN1 & Down-regulation & Suppressor & $\mathrm{HCC}$ & Progression & 19 \\
\hline 9 & hsa_circRNA_103809 & Down-regulation & Suppressor & $\mathrm{CRC}$ & Progression & 28 \\
\hline 10 & hsa_circRNA_104700 & Down-regulation & Suppressor & $\mathrm{CRC}$ & Progression & 28 \\
\hline 11 & hsa_circ_104423 & Down-regulation & Suppressor & $\mathrm{GC}$ & Recurrence & 25 \\
\hline 12 & hsa_circ_104916 & Down-regulation & Suppressor & GC & Recurrence & 25 \\
\hline 13 & hsa_circ_100269 & Down-regulation & Suppressor & $\mathrm{GC}$ & Recurrence & 25 \\
\hline 14 & hsa_circ_0005075 & Up-regulation & Oncogene & $\mathrm{HCC}$ & Growth & 21 \\
\hline 15 & circPVT1 & Up-regulation & Oncogene & $\mathrm{GC}$ & Proliferation & 30 \\
\hline 16 & ciRS7 (Cdr1as) & Up-regulation & Oncogene & $\mathrm{HCC}$ & Progression & 12 \\
\hline 17 & hsa_circRNA_101308 & Up-regulation & Oncogene & $\mathrm{GC}$ & Recurrence & 25 \\
\hline 18 & ciRS-7 - A & Up-regulation & Oncogene & $\mathrm{CRC}$ & Progression & 18 \\
\hline 19 & hsa_circRNA_100876 & Up-regulation & Oncogene & NSCLC & $\begin{array}{c}\text { Growth/Progression/ } \\
\text { Metastasis }\end{array}$ & 27 \\
\hline 20 & hsa_circ_100269 & Down-regulation & Suppressor & GC & Growth/Recurrence & 33 \\
\hline 21 & $\begin{array}{c}\text { circMTO1 }(\mathrm{hsa} \\
\text { circRNA_0007874/hsa } \\
\text { circRNA_104135) }\end{array}$ & Down-regulation & Suppressor & $\mathrm{HCC}$ & $\begin{array}{l}\text { Progression/Invasion/ } \\
\text { Growth }\end{array}$ & 42 \\
\hline 22 & circRNA-MYLK & Up-regulation & Oncogene & $\mathrm{BC}$ & Growth/Metastasis & 31 \\
\hline 23 & circRNA_100338 & Up-regulation & Oncogene & $\mathrm{HCC}$ & Metastasis & 40 \\
\hline 24 & hsa_circ_0003570 & Down-regulation & Suppressor & $\mathrm{HCC}$ & $\begin{array}{l}\text { Differentiation/ } \\
\text { Invasion }\end{array}$ & 39 \\
\hline 25 & Hsa_circ_0014717 & Down-regulation & Suppressor & $\mathrm{GC}$ & $\begin{array}{l}\text { Development/ } \\
\text { Progression }\end{array}$ & 37 \\
\hline 26 & hsa_circ_0013958 & Up-regulation & Oncogene & LAC & Invasion & 34 \\
\hline 27 & hsa_circ_100219 & Down-regulation & Suppressor & $\begin{array}{l}\text { Breast } \\
\text { Cancer }\end{array}$ & $\begin{array}{l}\text { Occurrence/ } \\
\text { Progression }\end{array}$ & 36 \\
\hline 28 & $\begin{array}{l}\text { hsa_circ_100219,hsa } \\
\text { circ_006054,hsa_circ_406697 }\end{array}$ & Down-regulation & Suppressor & $\begin{array}{l}\text { Breast } \\
\text { Cancer }\end{array}$ & $\begin{array}{l}\text { Occurrence/ } \\
\text { Progression }\end{array}$ & 36 \\
\hline 29 & hsa_circ_0006633 & Down-regulation & Suppressor & $\mathrm{GC}$ & Metastasis & 35 \\
\hline 30 & circCCDC66 & Up-regulation & Oncogene & $\mathrm{CRC}$ & $\begin{array}{l}\text { proliferation/migration/ } \\
\text { metastasis }\end{array}$ & 38 \\
\hline 31 & ciRS-7 & Up-regulation & Oncogene & $\mathrm{GC}$ & Growth/Metastasis & 32 \\
\hline 32 & circ-ITCH & Down-regulation & Suppressor & $\mathrm{HCC}$ & $\begin{array}{l}\text { Development/ } \\
\text { Progression }\end{array}$ & 41 \\
\hline
\end{tabular}

$\mathrm{GC}=$ Gastric Cancer; $\mathrm{HCC}=$ Hepatocellular Carcinoma; $\mathrm{CRC}=$ Colorectal Cancer; NSCLC $=$ Non-Small Cell Lung Cancer; $\mathrm{BC}=$ Bladder Cancer; LAC $=$ Lung Adenocarcinoma. 
Table 4: Results of subgroup and mete-regression analyses in the diagnostic meta-analysis

\begin{tabular}{|c|c|c|c|c|c|c|c|}
\hline Subgroup & $\begin{array}{c}\text { Number } \\
\text { of studies }\end{array}$ & $\operatorname{Se}(95 \% \mathrm{CI})$ & $\begin{array}{c}\text { Meta-regression } \\
(p \text {-value })\end{array}$ & $\mathrm{Sp}(95 \% \mathrm{CI})$ & $\begin{array}{c}\text { Meta-regression } \\
\text { (p-value) }\end{array}$ & $\operatorname{AUC}(95 \% \mathrm{CI})$ & $\begin{array}{c}\text { Meta-regression } \\
(p \text {-value })\end{array}$ \\
\hline Overall & 19 & $0.71(0.65-0.77)$ & & $0.77(0.72-0.81)$ & & $0.81(0.77-0.84)$ & \\
\hline Sample size & & & 0.857 & & 0.772 & & 0.672 \\
\hline$>100$ & 15 & $0.71(0.63-0.78)$ & & $0.76(0.70-0.80)$ & & $0.77(0.73-0.80)$ & \\
\hline$<100$ & 4 & $0.74(0.66-0.80)$ & & $0.84(0.75-0.90)$ & & $0.78(0.74-0.82)$ & \\
\hline Cancer type & & & 0.632 & & 0.964 & & 0.776 \\
\hline $\mathrm{GC}$ & 7 & $0.66(0.57-0.74)$ & & $0.80(0.72-0.85)$ & & $0.80(0.78-0.83)$ & \\
\hline $\mathrm{CRC}$ & 4 & $0.72(0.60-0.82)$ & & $0.67(0.58-0.76)$ & & $0.76(0.72-0.79)$ & \\
\hline $\mathrm{HCC}$ & 5 & $0.73(0.59-0.83)$ & & $0.79(0.72-0.85)$ & & $0.86(0.83-0.89)$ & \\
\hline
\end{tabular}

$\mathrm{GC}=$ Gastric Cancer; $\mathrm{CRC}=$ Colorectal Cancer; $\mathrm{HCC}=$ hepatocellular cancer; $\mathrm{AUC}=$ Area Under Curve; $\mathrm{Se}=$ Sensitivity; Sp=Specificity.

Meta-regression analysis for the subgroup have shown that the $P$ value was $>0.10$, suggesting no significant impact of subgroup on the pooled results.

\section{Sensitivity analysis and publication bias}

Sensitivity analysis for DFS/RFS and OS was also conducted. No remarkable change was observed when compared with previous results after removal of each study (Supplementary Figure 3).

Finally, we used Begg's funnel plot and Egger's test to evaluate the publication bias. Both the $P$ values for OS and DFS/RFS were 0.915 and 0.130 , respectively, suggesting no significant publication bias exists in the prognostic meta-analysis (Supplementary Figure 3).

\section{DISCUSSION}

Accumulating investigations have demonstrated aberrant circRNAs expression may play critical roles in cell proliferation, metastasis and recurrence of cancer. It has also been proven that circRNAs are expressed constantly in tissue, blood and tissue fluid [43]. Therefore, circRNAs may have the potential to be superior biomarkers for cancer diagnosis, prognosis and therapeutic estimate [6]. Recently, numerous studies have been conducted to explore it using relative small sample size. In the present study we collected all the relevant articles published to date and performed a systematic review and meta-analysis on the association of circRNAs
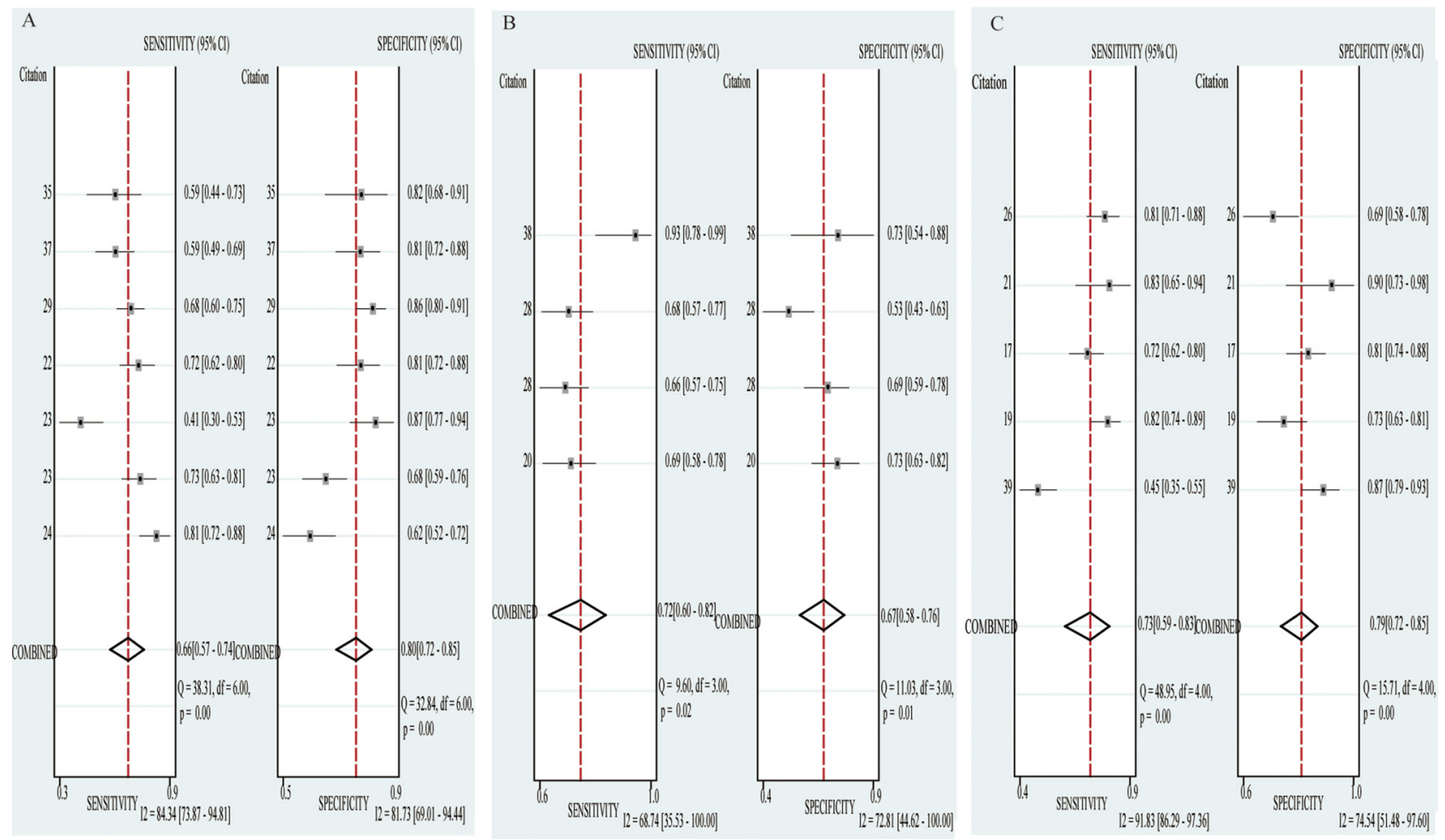

Figure 4: Forest plots of sensitivity and specificity of diagnostic articles in subgroup analysis. (A) Forest plots of GC subgroup. (B) Forest plots of CRC subgroup. (C) Forest plots of HCC subgroup. 
Table 5: Results of pooled HR(95\% CI) for prognostic articles

\begin{tabular}{ccc}
\hline All cancers & OS & DFS $/$ RFS \\
\hline HR $(95 \%$ CI $)$ & $1.37(0.98-1.75)$ & $2.28(0.77-3.79)$ \\
Heterogeneity, $P$ value & $99.2 \%, P=0.000$ & $99.1 \%, P=0.000$ \\
Pubbias $P$ value & 0.917 & 0.130 \\
Model & Random & Random \\
N & 1490 & 401 \\
Study Number & 11 & 4 \\
\hline
\end{tabular}

$\mathrm{HR}=$ hazard ratio; $\mathrm{CI}$ = confidence interval; OS=Overall Survival; DFS=Disease Free Survival; RFS=Recurrence Free Survival.

expression with cancer diagnosis and prognosis for the first time expecting to get relatively clear conclusions on whether circRNAs have the potential to be biomarkers for diagnosis and prognosis of cancer.

In this study, 18 circRNAs were related to cancer diagnosis, including 3 up-regulated circRNA (hsa circ_0005075, hsa_circ_0013958, circCCDC66) and 15 down-regulated circRNAs (hsa_circ_002059, hsa circ_001988, hsa_circ_0001649, hsa_circ_0000190, hsa_circ_0004018, hsa_circ_0001895, circZKSCAN1, hsa_circ_103809, hsa_circ_104700, hsa_circ_003570, hsa_circ_14717, hsa_circ_100219, hsa_circ_006054, hsa_circ_406697, hsa_circ_006633). It is widely believed that circRNAs are with cancer forewarning function. For example, hsa_circ_0000190 [23] and hsa_circ_0002059 [24] have been suggested to be capable of noninvasive markers for GC diagnosis; another research has indicated

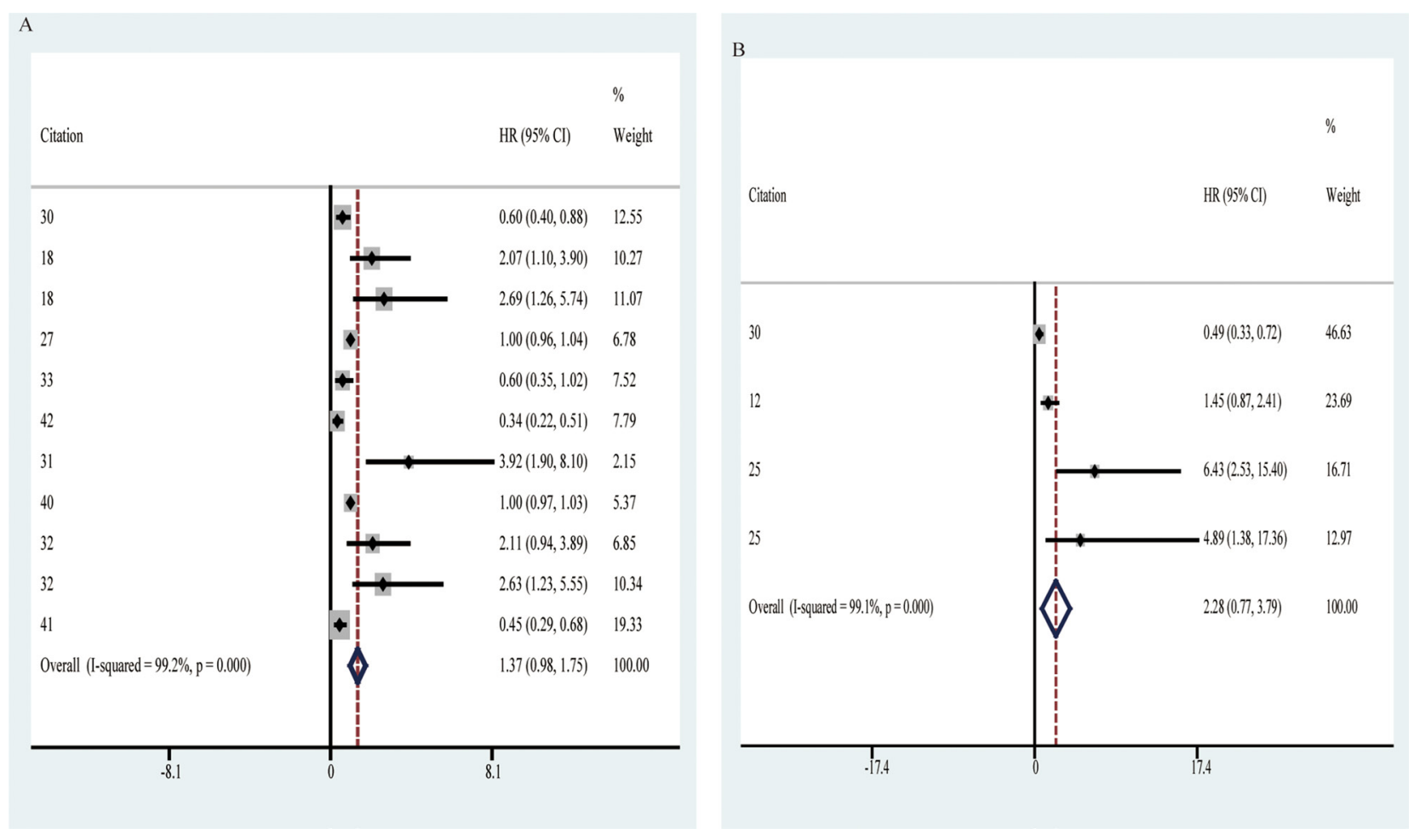

hsa_circ_0001649 as a potential diagnostic marker for HCC [26]. Our results showed that the overall sensitivity, specificity and AUC of multiple circRNAs were all more than $70 \%$, which were $0.71(0.65-0.77), 0.77$ $(0.72-0.81)$ and $0.81(0.77-0.84)$, respectively. Besides, the pooled DOR was 8.37 (6.14-11.39). A valid DOR should be greater than 1, and higher the value is, better the capability of testing discrimination could be obtained. The four above-mentioned parameters demonstrated that circRNAs expression might become promising biomarkers for cancer diagnosis. In stratified analysis, we also found circRNAs expression contributed a relatively high diagnostic specificity to GC and HCC, with the data were $0.80(0.72-0.85)$ and $0.79(0.72-0.85)$, suggesting the studied circRNAs might play important roles in the genesis and development of HCC. It has been reported that circZKSCAN1 can inhibit HCC cell proliferation,

Figure 5: Forest plots of pooled HR (95\% CI) of prognostic articles. (A) Pooled HR (95\% CI) of OS. (B) Pooled HR (95\% CI) of DFS/RFS. 
Table 6: Results of subgroup and mete-regression analyses in the prognostic meta-analysis of OS

\begin{tabular}{cccc}
\hline Subgroup & Number of studies & HR(95\% CI $)$ & Meta-regression $(\boldsymbol{p}$-value $)$ \\
\hline Function & & & 0.116 \\
Up-regulation & 8 & $1.85(1.26-2.44)$ & \\
Down-regulation & 3 & $0.46(0.32-0.59)$ & \\
\hline
\end{tabular}

$\mathrm{HR}=$ hazard ratio.

invasion and metastasis [19], and Cdrlas can promote microvascular infiltration of HCC [12]. Additionally, Chen et al have found hsa-circRNA-000190 in plasma is competent for early GC diagnosis [23]. Therefore, circRNAs could be applied to initial screening for cancer patients, which are beneficial for the improvement of their survival and life quality. Further investigations with larger number of samples are needed to validate these results and to promote clinical application of circRNAs as noninvasive biomarkers for cancer diagnosis.

Twelve prognosis-related circRNAs were involved in the meta-analysis, in which 6 were up regulated (circPVT1, ciRS-7, hsa_circ_101308, hsa_circ_100876, circRNAMYLK, circRNA_104135) and 6 were down regulated (hsa_circ 104423, hsa_circ_104916, hsa_circ_100269, hsa_circ_0007874, hsa_cir_104135, circ-ITCH). A circRNAs combination was found to be associated with poor prognosis for GC patients, containing the three down-regulated circRNAs and one up-regulated circRNA, hsa circ 101308. And another circRNAs combination including hsa_circ_0007874 and hsa_circ_104135 was related to more benign prognosis for $\mathrm{HCC}$ patients. Apart from them, the up-regulation of ciRS-7, hsa circ_100876, circRNA-MYLK, circRNA 100338 was also suggested poor prognosis, while circPVT1, hsa_circ_100269, hsa circ_0007874, hsa_circ_104135 and circ-ITCH indicated a better outcome. Generally speaking, oncogenes can elevate the susceptibility to cancer and confer to poor survival. However, some molecules were malignant could lead to better prognosis or higher sensitivity to chemotherapy [44], which was just demonstrated on circPVT1 in our study. Actually, it remains controversial whether circRNAs could serve as prognostic markers for OS or DFS/RFS. Weng et al found ciRS-7-A expression was associated with a worse OS of colorectal cancer [18]; while Jie Chen et al reported that circPVT1 contributed better OS to GC patients [30]. Similar phenomenon could also be discovered in the investigations about DFS/RFS [25; Chen, 2017 \#44]. In our stratified analysis, we found that the overall $\mathrm{HR}(95 \% \mathrm{CI})$ were $1.85(1.26,2.44)$ and $0.46(0.32,0.59)$ for up-regulated circRNAs and downregulated circRNAs, respectively, suggesting that the up-

\begin{tabular}{|c|c|c|c|}
\hline Citation & & $\operatorname{HR}(95 \% \mathrm{CI})$ & $\begin{array}{l}\% \\
\text { Weight }\end{array}$ \\
\hline \multicolumn{4}{|l|}{ 1. Up-regulated } \\
\hline 30 & $\leftarrow$ & $0.60(0.40,0.88)$ & 12.55 \\
\hline 18 & & $2.07(1.10,3.90)$ & 10.27 \\
\hline 18 & & $2.69(1.26,5.74)$ & 11.07 \\
\hline 27 & & $1.00(0.96,1.04)$ & 6.78 \\
\hline 31 & & $3.92(1.90,8.10)$ & 2.15 \\
\hline 40 & $\bullet$ & $1.00(0.97,1.03)$ & 5.37 \\
\hline 32 & & $2.11(0.94,3.89)$ & 6.85 \\
\hline 32 & & $2.63(1.23,5.55)$ & 10.34 \\
\hline Subtotal $(\mathrm{I}-\mathrm{squared}=99.9 \%, \mathrm{p}=0.000)$ & & $1.85(1.26,2.44)$ & 65.37 \\
\hline \multirow{2}{*}{\multicolumn{4}{|c|}{ 2. Down-regulated }} \\
\hline & & & \\
\hline 33 & 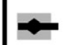 & $0.60(0.35,1.02)$ & 7.52 \\
\hline 42 & - & $0.34(0.22,0.51)$ & 7.79 \\
\hline 41 & $\sim$ & $0.45(0.29,0.68)$ & 19.33 \\
\hline Subtotal $(\mathrm{I}-\mathrm{squared}=38.2 \%, \mathrm{p}=0.198)$ & 4 & $0.46(0.32,0.59)$ & 34.63 \\
\hline Overall $(\mathrm{I}$-squared $=99.2 \%, \mathrm{p}=0.000)$ & & $1.37(0.98,1.75)$ & 100.00 \\
\hline${ }_{-8.1}^{T}$ & & & \\
\hline
\end{tabular}

Figure 6: Forest plot of pooled HR $(95 \% \mathrm{CI})$ of $\mathrm{OS}$ in up-regulated group and down-regulated group. 
regulated circRNAs can predict poor cancer prognosis and the down-regulated circRNAs may play the role of better cancer prognosis predictor. Notably, the prospects of circRNAs for clinical application will be quite broad if they are prognostic markers for cancer. Due to the stable expression of circRNAs in various body fluids, they could provide more effective information for clinical prediction in the perioperative period when compared with clinical parameters such as tumor size and clinicopathologic stage. Further large-scale investigations are needed to identify novel circRNAs and to comprehensively and objectively explore their clinical roles as promising biomarkers for cancer prognosis.

Several limitations should be acknowledged. First, all the samples in our study were selected from Asian population and the detection method for circRNAs expression was major in qRT-PCR. Single sample source and technology might mask the possible impacts of ethnicity and experimental methods on the results. Second, some literatures was not successfully extracted due to the no response of the investigators, which would produce some bias for the selection of the recruitment. Moreover, the sample size involved in the meta-analysis was still relatively small limited by few available articles to date.

In summary, as a type of stably expressed molecules, circRNAs could be promising biomarkers for diagnosis and prognosis of cancers. More association studies focusing on circRNAs expression with cancer are needed to further explore the practical values of circRNAs expression on clinical diagnosis and treatment.

\section{MATERIALS AND METHODS}

This study was carried out on the basis of Preferred Reporting Items for Systematic Reviews and Metaanalysis (PRISMA) [45].

\section{Search strategy}

A literature search of PubMed and Web of Science was performed for studies related to the association of circRNAs with cancer diagnosis or (and) prognosis up to September 10th, 2017, using the following key words: "circRNA cancer", "circRNA carcinoma", "circRNA tumor", "circRNA neoplasm”, "circularRNA cancer", "circularRNA carcinoma", "circularRNA tumor", "circularRNA neoplasm".

\section{Selection criteria}

Two reviewers (Hanxi Ding and Qian Xu) evaluated the eligibility of retrieved articles independently. All selected studies met the following criteria: (1) Cases were histopathologically diagnosed as cancer; (2) Information of control groups was available; (3) CircRNAs were used for cancer diagnosis or prognosis; (4) The effect indicators contained AUC, sensitivity, specificity or OS, DFS, RFS,
HR and 95\% CI; (5) Data was sufficient for quantitative analysis. The exclusion criteria were: (1) Duplicate studies; (2) Reviews; (3) Not related to human or cancer; (4) Irrelevant to the study subject; (5) Insufficient data for quantitative analysis. Two reviewers reached consensus regarding all items.

\section{Data extraction}

Two investigators (Hanxi Ding and Qian $\mathrm{Xu}$ ) independently extracted the data according to critical criteria. The following information was obtained from each article: first author's name, publication year, origin country and ethnicity, circRNAs' name, cancer type and stage, total number of cases, sample source, and detection method. Diagnostic indicators included sensitivity, specificity and AUC; Prognostic indicators were survival and HR with $95 \%$ CI for DFS or OS. When HRs with 95CIs were not presented in the study, they were extracted from Kaplan-Meier survival curves using a method introduced by Tierney et al [46].

\section{Quality assessment}

Quality assessment of diagnostic accuracy studies-2 (QUADAS-2) was employed to evaluate the quality of enrolled studies. Prognostic studies quality was assessed based on the Newcastle-Ottawa Scale [47].

\section{Statistical analysis}

All analyses were conducted using Stata software, version 11.0. $P<0.05$ was considered as statistically significant.

Sensitivity, specificity and AUC were involved in the diagnostic meta-analysis. The pooled parameters were all estimated by continuous meta-analysis model. The area under summary receiver operator characteristic curve (SROC) was calculated to evaluate the diagnostic efficacy. Inter-study heterogeneity was examined with the $\mathrm{I}^{2}$ statistic [48]. To explore the possible source of heterogeneity, stratified analysis based on cancer type and sample size as well as meta-regression were performed [49]. Deek's funnel plot was employed to assess the publication bias [50]. Sensitivity analysis was also conducted.

In the prognostic meta-analysis, the pooled OR with 95\% CI was calculated to evaluate the association between circRNAs expression and survival of cancer patients in both fixed-effect and random-effect models. Cochran's $\mathrm{Q}$ test and $\mathrm{I}^{2}$ statistic were used to judge the inter-study heterogeneity [51]. We pooled the results using fixedeffect model when $P>0.10$ and $\mathrm{I}^{2}<50 \%$, suggesting an absent heterogeneity [52]; otherwise the random-effect model would be chose. Begg's funnel plot was employed to assess the publication bias [53]. Sensitivity analysis was also conducted. 


\section{Abbreviations}

GC, Gastric Cancer; HCC, Hepatocellular Carcinoma; CRC, Colorectal Cancer; NSCLC, NonSmall Cell Lung Cancer; AUC, Area Under Curve; ROC, receiver operating characteristic curve; Se, Sensitivity; Sp, Specificity; DFS, Disease Free Survival; RFS, Recurrence Free Survival; OS, Overall Survival.

\section{Author contributions}

Yuan Yuan conceived and designed this study. Han-xi Ding and Qian Xu was responsible for the data extraction and performed data interpretation. Han-xi Ding wrote the paper. Qian $\mathrm{Xu}$, Zhi Lv and Yuan Yuan revised the manuscript. All authors read and approved the final manuscript, and do not have a commercial or other association that might pose a conflict of interest.

\section{CONFLICTS OF INTEREST}

The authors declare that they have no conflicts of interest.

\section{FUNDING}

This manuscript is supported by the National Natural Science Foundation (81772987), Natural Science Foundation of Liaoning Province, China (20170540987) and the Outstanding Youth Foundation of China Medical University (YQ20170006).

\section{REFERENCES}

1. Li J, Yang J, Zhou P, Le Y, Zhou C, Wang S, Xu D, Lin HK, Gong Z. Circular RNAs in cancer: novel insights into origins, properties, functions and implications. Am J Cancer Res. 2015; 5:472-80.

2. Hou LD, Zhang J. Circular RNAs: An emerging type of RNA in cancer. Int J Immunopathol Pharmacol. 2017; 30:1-6.

3. Wang Y, Mo Y, Gong Z, Yang X, Yang M, Zhang S, Xiong F, Xiang B, Zhou M, Liao Q, Zhang W, Li X, Li X, et al. Circular RNAs in human cancer. Dis Markers. 2017; 16:25.

4. Suzuki H, Tsukahara T. A view of pre-mRNA splicing from RNase R resistant RNAs. Int J Mol Sci. 2014; 15:9331-42.

5. Chen I, Chen CY, Chuang TJ. Biogenesis, identification, and function of exonic circular RNAs. Wiley Interdiscip Rev RNA. 2015; 6:563-79.

6. Dong Y, He D, Peng Z, Peng W, Shi W, Wang J, Li B, Zhang C, Duan C. Circular RNAs in cancer: an emerging key player. J Hematol Oncol. 2017; 10:2.

7. Hansen TB, Jensen TI, Clausen BH, Bramsen JB, Finsen B, Damgaard CK, Kjems J. Natural RNA circles function as efficient microRNA sponges. Nature. 2013; 495:384-8.
8. Granados-Riveron JT, Aquino-Jarquin G. The complexity of the translation ability of circRNAs. Biochim Biophys Acta. 2016; 1859:1245-51.

9. Xue J, Liu Y, Luo F, Lu X, Xu H, Liu X, Lu L, Yang Q, Chen C, Fan W, Liu Q. Circ100284, via miR-217 regulation of EZH2, is involved in the arsenite-accelerated cell cycle of human keratinocytes in carcinogenesis. Biochim Biophys Acta. 2017; 1863:753-63.

10. Li F, Zhang L, Li W, Deng J, Zheng J, An M, Lu J, Zhou Y. Circular RNA ITCH has inhibitory effect on ESCC by suppressing the Wnt/beta-catenin pathway. Oncotarget. 2015; 6:6001-13. https://doi.org/10.18632/oncotarget.3469.

11. Yang W, Du WW, Li X, Yee AJ, Yang BB. Foxo3 activity promoted by non-coding effects of circular RNA and Foxo3 pseudogene in the inhibition of tumor growth and angiogenesis. Oncogene. 2016; 35:3919-31.

12. Xu L, Zhang M, Zheng X, Yi P, Lan C, Xu M. The circular RNA ciRS-7 (Cdrlas) acts as a risk factor of hepatic microvascular invasion in hepatocellular carcinoma. J Cancer Res Clin Oncol. 2017; 143:17-27.

13. Memczak S, Jens M, Elefsinioti A, Torti F, Krueger J, Rybak A, Maier L, Mackowiak SD, Gregersen LH, Munschauer M, Loewer A, Ziebold U, Landthaler M, et al. Circular RNAs are a large class of animal RNAs with regulatory potency. Nature. 2013; 495:333-8.

14. Salzman J, Gawad C, Wang PL, Lacayo N, Brown PO. Circular RNAs are the predominant transcript isoform from hundreds of human genes in diverse cell types. PLoS One. 2012; 7:e30733.

15. Lasda E, Parker R. Circular RNAs Co-Precipitate with Extracellular Vesicles: A Possible Mechanism for circRNA Clearance. PLoS One. 2016; 11:e0148407.

16. Jeck WR, Sorrentino JA, Wang K, Slevin MK, Burd CE, Liu J, Marzluff WF, Sharpless NE. Circular RNAs are abundant, conserved, and associated with ALU repeats. RNA. 2013; 19:141-57.

17. Fu L, Yao T, Chen Q, Mo X, Hu Y, Guo J. Screening differential circular RNA expression profiles reveals hsa circ_0004018 is associated with hepatocellular carcinoma. Oncotarget. 2017; 8:58405-58416. https://doi.org/10.18632/ oncotarget. 16881 .

18. Weng W, Wei Q, Toden S, Yoshida K, Nagasaka T, Fujiwara T, Cai S, Qin H, Ma Y, Goel A. Circular RNA ciRS-7 - A promising prognostic biomarker and a potential therapeutic target in colorectal cancer. Clin Cancer Res. 2017; 23:39183928.

19. Yao Z, Luo J, Hu K, Lin J, Huang H, Wang Q, Zhang P, Xiong Z, He C, Huang Z, Liu B, Yang Y. ZKSCAN1 gene and its related circular RNA (circZKSCAN1) both inhibit hepatocellular carcinoma cell growth, migration, and invasion but through different signaling pathways. Mol Oncol. 2017; 11:422-37.

20. Wang X, Zhang Y, Huang L, Zhang J, Pan F, Li B, Yan Y, Jia B, Liu H, Li S, Zheng W. Decreased expression of hsa_circ_001988 in colorectal cancer and its clinical significances. Int J Clin Exp Pathol. 2015; 8:16020-5. 
21. Thomas E, Shang X, Li G, Liu H, Li T, Liu J, Zhao Q, Wang C. Comprehensive Circular RNA Profiling Reveals That hsa_circ_0005075, a New Circular RNA Biomarker, Is Involved in Hepatocellular Crcinoma Development. J Immunol. 2016; 95:e3811.

22. Li WH, Song YC, Zhang H, Zhou ZJ, Xie X, Zeng QN, Guo K, Wang T, Xia P, Chang DM. Decreased Expression of Hsa_circ_00001649 in Gastric Cancer and Its Clinical Significance. Dis Markers. 2017; 2017:4587698.

23. Chen S, Li T, Zhao Q, Xiao B, Guo J. Using circular RNA hsa_circ_0000190 as a new biomarker in the diagnosis of gastric cancer. Clin Chim Acta. 2017; 466:167-71.

24. Li P, Chen S, Chen H, Mo X, Li T, Shao Y, Xiao B, Guo J. Using circular RNA as a novel type of biomarker in the screening of gastric cancer. Clin Chim Acta. 2015; 444:132-6.

25. Zhang Y, Li J, Yu J, Liu H, Shen Z, Ye G, Mou T, Qi $\mathrm{X}$, Li G. Circular RNAs signature predicts the early recurrence of stage III gastric cancer after radical surgery. Oncotarget. 2017; 8:22936-43. https://doi.org/10.18632/ oncotarget. 15228 .

26. Qin M, Liu G, Huo X, Tao X, Sun X, Ge Z, Yang J, Fan J, Liu L, Qin W. Hsa_circ_0001649: A circular RNA and potential novel biomarker for hepatocellular carcinoma. Cancer Biomark. 2016; 16:161-9.

27. Yao JT, Zhao SH, Liu QP, Lv MQ, Zhou DX, Liao ZJ, Nan KJ. Over-expression of CircRNA_100876 in non-small cell lung cancer and its prognostic value. Pathol Res Pract. 2017; 213:453-456.

28. Zhang P, Zuo Z, Shang W, Wu A, Bi R, Wu J, Li S, Sun X, Jiang L. Identification of differentially expressed circular RNAs in human colorectal cancer. Tumour Biol. 2017; 39:1010428317694546.

29. Shao Y, Chen L, Lu R, Zhang X, Xiao B, Ye G, Guo J. Decreased expression of hsa_circ_0001895 in human gastric cancer and its clinical significances. Tumour Biol. 2017; 39:1010428317699125.

30. Chen J, Li Y, Zheng Q, Bao C, He J, Chen B, Lyu D, Zheng B, Xu Y, Long Z, Zhou Y, Zhu H, Wang Y, et al. Circular RNA profile identifies circPVT1 as a proliferative factor and prognostic marker in gastric cancer. Cancer Lett. 2017; 388:208-19.

31. Zhong Z, Huang M, Lv M, He Y, Duan C, Zhang L, Chen J. Circular RNA MYLK as a competing endogenous RNA promotes bladder cancer progression through modulating VEGFA/VEGFR2 signaling pathway. Cancer Lett. 2017; 403:305-17. https://doi.org/10.1016/j.canlet.2017.06.027.

32. Pan H, Li T, Jiang Y, Pan C, Ding Y, Huang Z, Yu H, Kong D. Overexpression of Circular RNA ciRS-7 Abrogates the Tumor Suppressive Effect of miR-7 on Gastric Cancer via PTEN/PI3K/AKT Signaling Pathway. J Cell Biochem. 2017; 119:440-446.

33. Zhang Y, Liu H, Li W, Yu J, Li J, Shen Z, Ye G, Qi X, Li G. CircRNA_100269 is downregulated in gastric cancer and suppresses tumor cell growth by targeting miR-630. Aging
(Albany NY). 2017; 9:1585-94. https://doi.org/10.18632/ aging. 101254.

34. Zhu X, Wang X, Wei S, Chen Y, Chen Y, Fan X, Han S, Wu G. hsa_circ_0013958: a circular RNA and potential novel biomarker for lung adenocarcinoma. FEBS J. 2017; 284:2170-82.

35. Lu R, Shao Y, Ye G, Xiao B, Guo J. Low expression of hsa_circ_0006633 in human gastric cancer and its clinical significances. Tumour Biol. 2017; 39:1010428317704175.

36. Lu L, Sun J, Shi P, Kong W, Xu K, He B, Zhang S, Wang J. Identification of circular RNAs as a promising new class of diagnostic biomarkers for human breast cancer. Oncotarget. 2017; 8:44096-107. https://doi.org/10.18632/ oncotarget. 17307.

37. Shao Y, Li J, Lu R, Li T, Yang Y, Xiao B, Guo J. Global circular RNA expression profile of human gastric cancer and its clinical significance. Cancer Med. 2017; 6:1173-80.

38. Hsiao KY, Lin YC, Gupta SK, Chang N, Yen L, Sun HS, Tsai SJ. Noncoding Effects of Circular RNA CCDC66 Promote Colon Cancer Growth and Metastasis. Cancer Res. 2017; 77:2339-50.

39. Fu L, Wu S, Yao T, Chen Q, Xie Y, Ying S, Chen Z, Xiao B, Hu Y. Decreased expression of hsa_circ_0003570 in hepatocellular carcinoma and its clinical significance. J Clin Lab Anal. 2017 May 11. [Epub ahead of print].

40. Huang XY, Huang ZL, Xu YH, Zheng Q, Chen Z, Song W, Zhou J, Tang ZY, Huang XY. Comprehensive circular RNA profiling reveals the regulatory role of the circRNA-100338/ miR-141-3p pathway in hepatitis B-related hepatocellular carcinoma. Sci Rep. 2017; 7:5428.

41. Guo W, Zhang J, Zhang D, Cao S, Li G, Zhang S, Wang Z, Wen P, Yang H, Shi X, Pan J, Ye H. Polymorphisms and expression pattern of circular RNA circ-ITCH contributes to the carcinogenesis of hepatocellular carcinoma. Oncotarget. 2017; 8:48169-77. https://doi.org/10.18632/oncotarget.18327.

42. Han D, Li J, Wang H, Su X, Hou J, Gu Y, Qian C, Lin Y, Liu X, Huang M, Li N, Zhou W, Yu Y, et al. Circular RNA circMTO1 acts as the sponge of microRNA-9 to suppress hepatocellular carcinoma progression. Hepatology. 2017; 66:1151-1164.

43. Wang F, Nazarali AJ, Ji S. Circular RNAs as potential biomarkers for cancer diagnosis and therapy. Am J Cancer Res. 2016; 6:1167-76.

44. Liu X, Liu J, Qiu H, Kong P, Chen S, Li W, Zhan Y, Li Y, Chen Y, Zhou Z, Xu D, Sun X. Prognostic significance of Epstein-Barr virus infection in gastric cancer: a metaanalysis. BMC Cancer. 2015; 15:782.

45. Moher D, Liberati A, Tetzlaff J, Altman DG. Preferred reporting items for systematic reviews and meta-analyses: the PRISMA statement. Int J Surg. 2010; 8:336-41.

46. Tierney JF, Stewart LA, Ghersi D, Burdett S, Sydes MR. Response to: Practical methods for incorporating summary time-to-event data into meta. Authors' reply. Trials. 2013; 14:391. 
47. Stang A. Critical evaluation of the Newcastle-Ottawa scale for the assessment of the quality of nonrandomized studies in meta-analyses. Eur J Epidemiol. 2010; 25:603-5.

48. Higgins JP, Thompson SG, Deeks JJ, Altman DG. Measuring inconsistency in meta-analyses. Bmj. 2003; 327:557-60.

49. Song F, Sheldon TA, Sutton AJ, Abrams KR, Jones DR. Methods for exploring heterogeneity in meta-analysis. Eval Health Prof. 2001; 24:126-51.

50. Deeks JJ, Macaskill P, Irwig L. The performance of tests of publication bias and other sample size effects in systematic reviews of diagnostic test accuracy was assessed. J Clin Epidemiol. 2005; 58:882-93.

51. Higgins JP, Thompson SG. Quantifying heterogeneity in a meta-analysis. Stat Med. 2002; 21:1539-58.

52. Biggerstaff BJ, Tweedie RL. Incorporating variability in estimates of heterogeneity in the random effects model in meta-analysis. Stat Med. 1997; 16:753-68.

53. Egger M, Davey Smith G, Schneider M, Minder C. Bias in meta-analysis detected by a simple, graphical test. Bmj. 1997; 315:629-34. 